\title{
A CMOS Tunable Transimpedance Amplifier
}

\author{
Huei-Yan Hwang, Jun-Chau Chien, Student Member, IEEE, Tai-Yuan Chen, and Liang-Hung Lu, Member, IEEE
}

\begin{abstract}
A tunable transimpedance amplifier (TIA) is presented in this letter. By incorporating a mechanism for gain and bandwidth tuning, the TIA can be adjusted to achieve optimum circuit performance with a lowest bit-error-rate (BER) for high-speed applications. The proposed circuit is implemented in a 0.18- $\mu \mathrm{m}$ CMOS process. Consuming a dc power of $34 \mathrm{~mW}$ from a 2.0-V supply voltage, the fabricated TIA exhibits a variable -3-dB bandwidth from 3.9 to $7.6 \mathrm{GHz}$ while maintaining a transimpedance gain of $52 \mathrm{~dB} \Omega$. With a $7.5-\mathrm{Gb} / \mathrm{s} 2^{31}-1$ pseudo-random bit sequence, the measured input sensitivity of the TIA is $-19 \mathrm{dBm}$ at a BER of $10^{-12}$.
\end{abstract}

Index Terms-Bit-error-rate (BER), input sensitivity, intersymbol interference (ISI), optical receiver, regulated cascode, total integrated noise, transimpedance amplifier (TIA), tunable bandwidth.

\section{INTRODUCTION}

$\mathbf{I}$ $\mathrm{N}$ THE receiver frontend of an optical-fiber communication system, transimpedance amplifiers (TIAs) are widely used as the first active building block to convert the photodiode current to an amplified voltage for data recovery. Since the system specifications such as sensitivity, speed, and signal-to-noise ratio are strongly influenced by the TIA, tremendous design efforts are required in determining the circuit parameters for optimum performance. Due to the inherently high speed and low noise characteristics, III-V compound semiconductor devices have been dominantly utilized to realize such amplifiers [1], [2]. With the continuous scaling of transistor feature size, fully integrated TIA designs using submicron CMOS technologies have attracted great attention due to the low implementation cost and high integration level. In recent years, CMOS TIAs with a bandwidth up to $20 \mathrm{GHz}$ [3]-[6] have been reported for optoelectronic integrated circuit (OEIC) applications.

In the design of a TIA, one of the most important specifications is the $-3-\mathrm{dB}$ bandwidth. Theoretically, the TIA bandwidth is chosen for the lowest bit-error-rate (BER) possible at a given data rate. Considering the effect of noise which is inevitable in all communication systems, it is desirable to minimize the bandwidth so as to reduce the total integrated noise within the passband. On the other hand, the limited bandwidth introduces intersymbol interference (ISI) in the random data, causing eye closure for the received signals. As a result, a design tradeoff is established between the integrated noise and ISI in determining

Manuscript received May 23, 2006; revised June 13, 2006. This work was supported in part by the National Science Council of Taiwan, R.O.C., under Grants 94-2220-E-002-026 and 94-2220-E-002-009, the National Chip Implementation Center (CIC), and the National Nano Device Laboratories (NDL).

The authors are with the Department of Electrical Engineering and Graduate Institute of Electronics Engineering, National Taiwan University, Taipei, Taiwan 10617, R.O.C. (e-mail: 1hlu@cc.ee.ntu.edu.tw).

Color versions of Figs. 2 and 4 are available at http://ieeexplore.ieee.org.

Digital Object Identifier 10.1109/LMWC.2006.885641 the amplifier bandwidth. A rule of thumb which is typically adopted for TIA designs is to use $70 \%$ of the required data rate as the $-3-\mathrm{dB}$ bandwidth. Since the effective noise strongly depends on the channel characteristics and the circuit design, it fails to provide an optimum performance in most cases. In this letter, a tunable CMOS TIA is proposed for high-speed applications. With the built-in tuning mechanism, the gain and bandwidth of the fabricated TIA can be adjusted to compensate for the process variation. Furthermore, an optimum operation can be reached between the integrated noise and ISI, achieving a low BER in practical circuit implementations.

In Section II, the circuit topology and operation principle of the proposed TIA are described. The experimental results of the tunable CMOS TIA are presented in Section III. Finally, a conclusion is provided in Section IV.

\section{PROPOSED TIA TOPOLOGY}

Fig. 1 shows the complete schematic of the proposed TIA with all onchip components. Instead of using a common-gate transistor, a regulated cascode stage $M_{1}-M_{3}$ is employed as the input current buffer to enhance the amplifier bandwidth while maintaining the required input impedance. The gain stage is composed of a source follower $M_{4}$ and cascode transistors $M_{5}-M_{6}$. The purpose of the source follower is to provide a dc level shift and better interstage impedance matching. On the other hand, the cascode stage is designed for a high voltage gain. In the proposed circuit topology, a voltage-current feedback provided by $R_{f}$ is included to reduce the effective impedance at the input and output nodes of the gain stage, pushing the poles of the amplifier to higher frequencies. Transistors $M_{7}$ and $M_{8}$ are used as the output buffer of the TIA. In order to achieve the required bandwidth for high-speed applications, inductive peaking technique is adopted by adding spiral inductors $L_{1}-L_{4}$ in series connection with the resistive loads for the individual stages. Based on the simulation results, a $25 \%$ increase in $-3-\mathrm{dB}$ bandwidth is observed due to the use of the inductive loads.

Based on the small-signal analysis, the transimpedance gain of the proposed TIA at lower frequencies can be expressed as

$$
\begin{aligned}
Z_{21} \equiv & \left|\frac{v_{\text {out }}}{i_{\text {in }}}\right| \\
= & \frac{g_{m 1} R_{1} R_{S}\left(1+g_{m 2} R_{2}\right)}{1+g_{m 1} R_{S}\left(1+g_{m 2} R_{2}\right)} \\
& \times \frac{g_{m 5} R_{3} R_{f}^{2}}{\left(R_{1}+R_{f}\right)\left(R_{3}+R_{f}\right)+g_{m 5} R_{1} R_{3} R_{f}} \\
& \times g_{m 8}\left(R_{4} \| R_{L}\right)
\end{aligned}
$$

where $R_{S}$ and $R_{L}$ represent the source and load resistance, respectively. Assuming that the values of $g_{m 2} R_{2}, g_{m 5} R_{3}$, and 


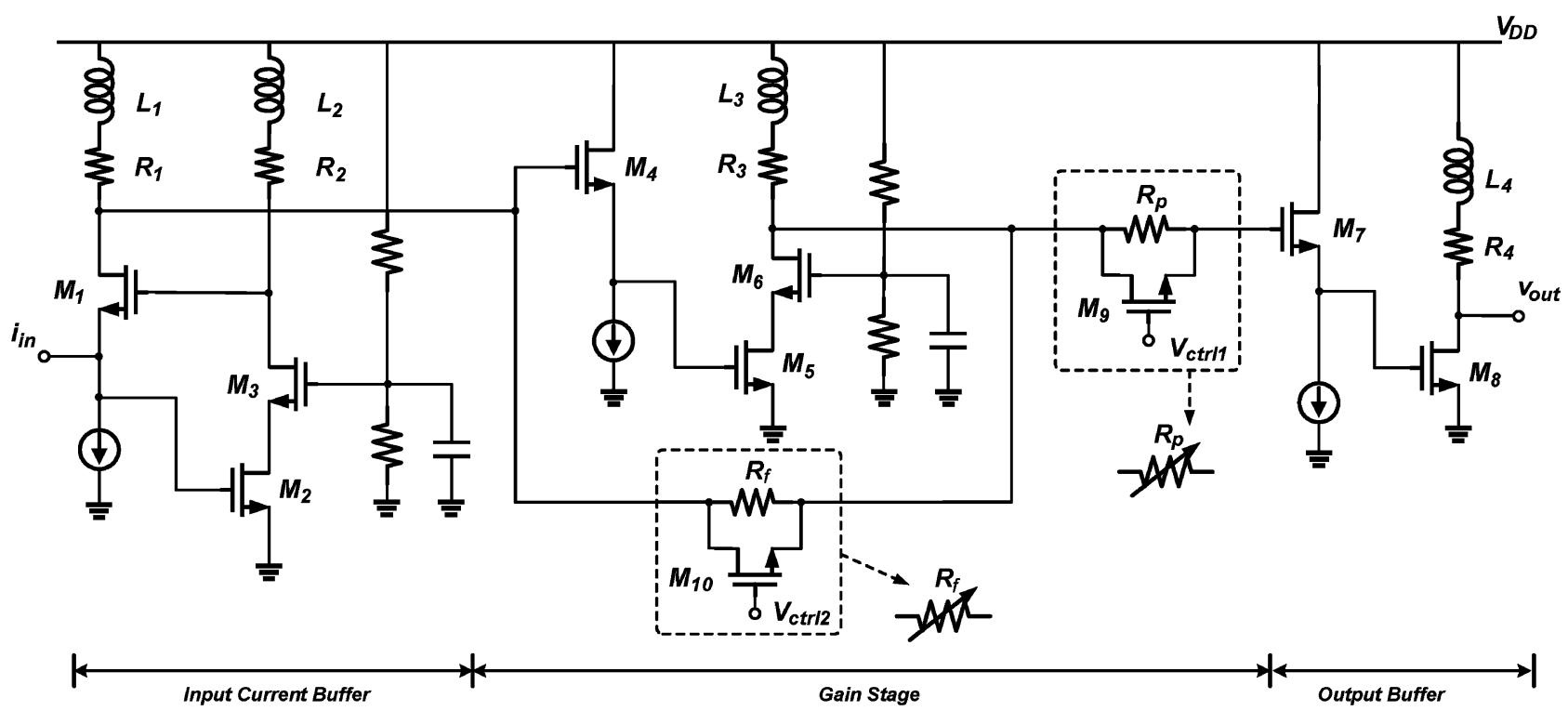

Fig. 1. Schematic of the proposed tunable TIA.

$g_{m 5} R_{f}$ are much larger than unity and the dominant pole is determined by the RC time constant at the drain of $M_{6}$, the $-3-\mathrm{dB}$ bandwidth of the TIA is approximated by

$$
\omega_{-3 \mathrm{~dB}}=\left(\frac{R_{3} C_{p}}{1+R_{1} / R_{f}+R_{1} R_{3} / R_{f}^{2}}+R_{p} C_{p}\right)^{-1}
$$

where $C_{p}$ is the equivalent capacitance at the gate of $M_{7}$.

The tuning mechanism of the TIA gain and bandwidth is provided by transistors $M_{9}$ and $M_{10}$, which are operated in the triode region as tunable resistors. As the controlled voltage $V_{c t r l 1}$ decreases, the effective resistance of $R_{p}$ increases, moving the dominant pole of the TIA toward a lower frequency. Hence, the bandwidth can be tuned over a very wide range while maintaining a constant transimpedance gain. Alternatively, simultaneous gain and bandwidth tuning can also be achieved to compensate for the process variation by adjusting the value of feedback resistance $R_{f}$ with the controlled voltage $V_{c t r l 2}$.

\section{EXPERIMENTAL RESULTS}

The proposed tunable TIA is designed and implemented in a $0.18-\mu \mathrm{m}$ CMOS process. Fig. 2 shows the die photograph of the fabricated circuit with a chip size of $0.84 \times 0.61 \mathrm{~mm}^{2}$ including the pad frame. On-wafer probing was performed to characterize the performance of the TIA.

With a supply voltage of $2.0 \mathrm{~V}$, the fabricated amplifier with the on-chip bias circuitry draws a dc current of $17 \mathrm{~mA}$. The small-signal characteristics of the TIA were evaluated by the $S$-parameter measurement. As the controlled voltage $\left(V_{c t r l 1}\right)$ varies, the tunable TIA exhibits a $-3-\mathrm{dB}$ bandwidth ranging from 3.9 to $7.6 \mathrm{GHz}$ while maintaining a transimpedance gain $\left(Z_{21}\right)$ of $52 \mathrm{~dB} \Omega$. The measured frequency responses, including the transimpedance gain and the group delay, of the TIA with various bandwidths are shown in Fig. 3 .

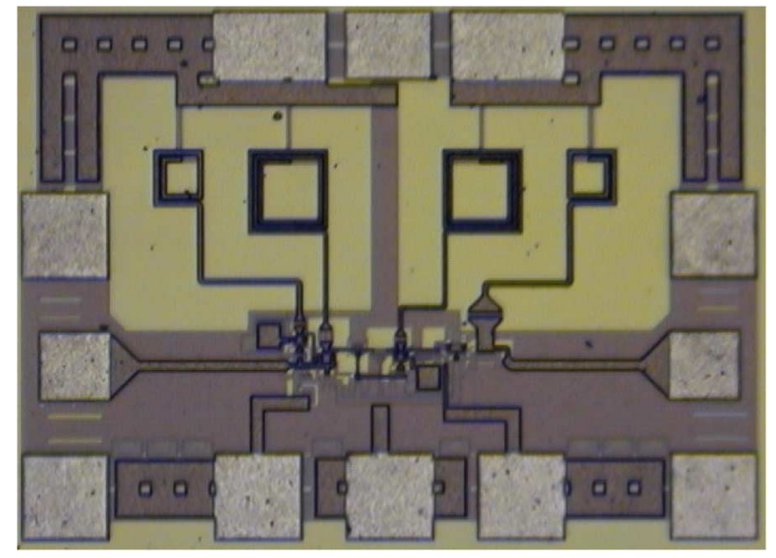

Fig. 2. Die photograph of the fabricated TIA.

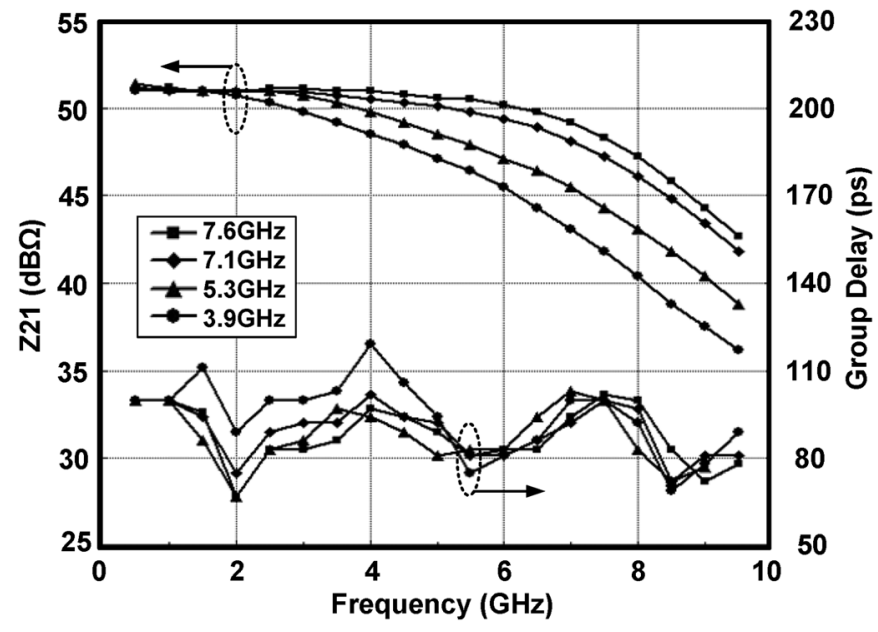

Fig. 3. Measured transimpedance gain and group delay of the TIA.

To characterize the optimum bandwidth of the TIA for highspeed operations, an Agilent 86130A BERT system and an Agilent $86100 \mathrm{C}$ oscilloscope are used for the eye diagram and 


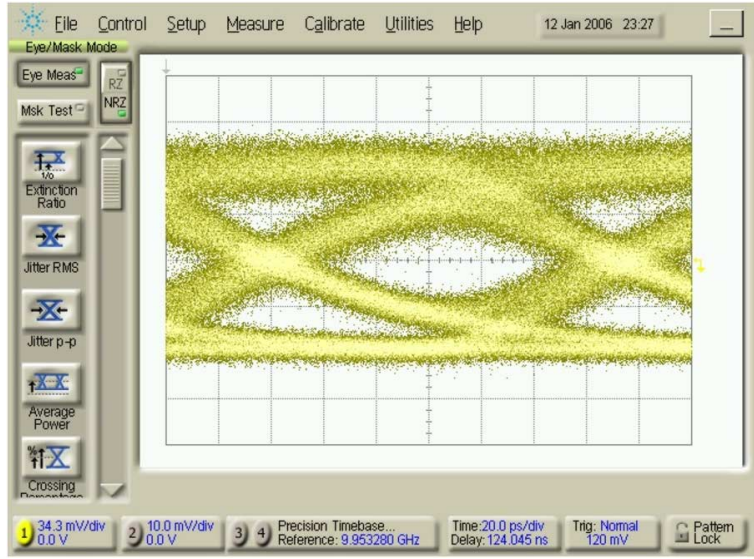

Fig. 4. Measured eye diagrams of the TIA with $7.5-\mathrm{Gb} / \mathrm{s} 2^{31}-1$ PRBS input. (horizontal scale: $20 \mathrm{ps} / \mathrm{div}$, vertical scale: $34.3 \mathrm{mv} / \mathrm{div}$ ).

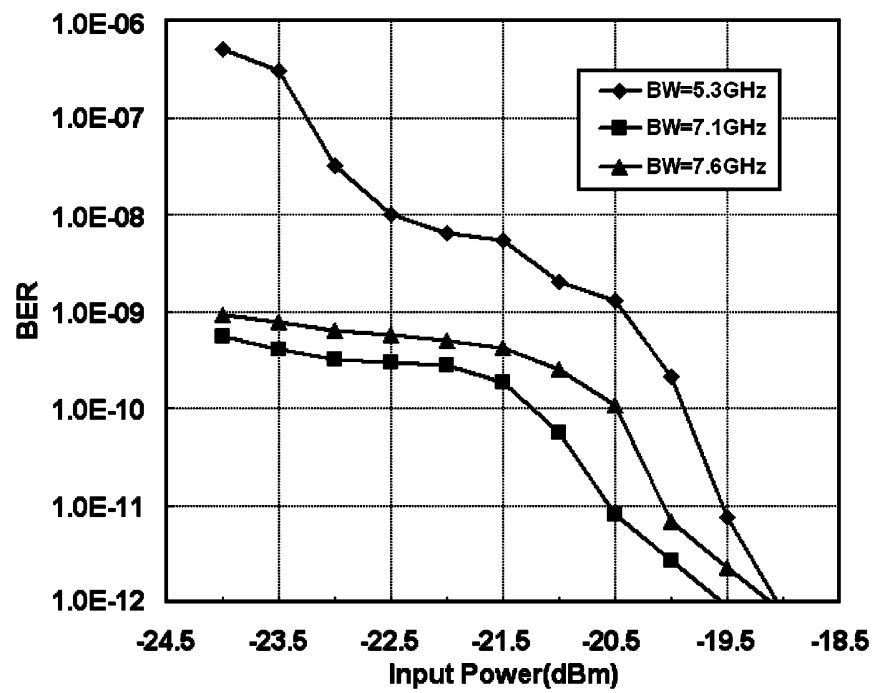

Fig. 5. Measured BER of the TIA with 7.5-Gb/s $2^{31}-1$ PRBS input.

BER measurement. For a $7.5-\mathrm{Gb} / \mathrm{s} 2^{31}-1$ pseudo-random bit sequence (PRBS) with an input power of $-21 \mathrm{dBm}$, the output eye diagram of the TIA operating at a bandwidth of $7.1 \mathrm{GHz}$ is shown in Fig. 4, indicating a vertical eye opening greater than $40 \mathrm{mV}$. Fig. 5 shows the measured BER of the TIA versus the input power. For an input data rate of $7.5 \mathrm{~Gb} / \mathrm{s}$, the proposed TIA exhibits a lowest BER with an optimum bandwidth of $7.1 \mathrm{GHz}$. The obtained input sensitivity for a BER of $10^{-12}$ is $-19 \mathrm{dBm}$. In order to evaluate the influences of the photodiode on the -3-dB bandwidth, the frequency responses of the TIA with an input capacitance of $250 \mathrm{fF}$ are shown in Fig. 6. It is observed that the bandwidth degradation manifests itself when the TIA is tuned for a maximum bandwidth. The performance of the tunable TIA is summarized in Table I.

\section{CONCLUSION}

A novel TIA circuit with tunable gain and -3-dB bandwidth is proposed. Using a standard $0.18-\mu \mathrm{m}$ CMOS process, the fully integrated TIA is realized for OEIC applications. By sweeping the controlled voltages, the fabricated circuit exhibits a band-

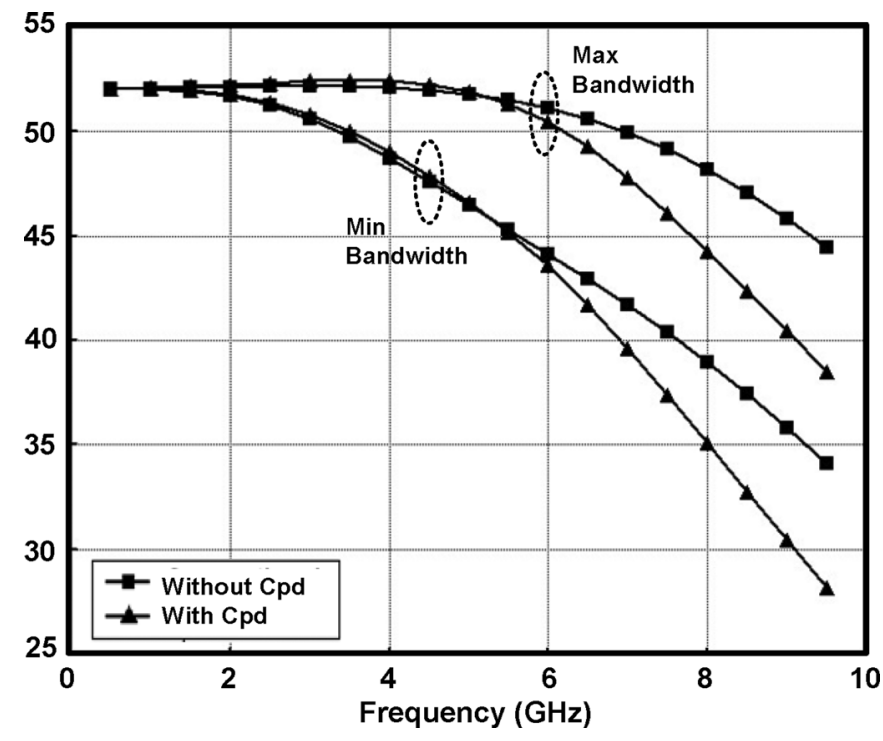

Fig. 6. Frequency responses of the tunable TIA with a $250-\mathrm{fF}$ photodiode capacitance $C_{p d}$.

TABLE I

Performance Summary of the Tunable Transimpedance Amplifier

\begin{tabular}{|c|c|}
\hline \hline Technology & $0.18-\mu \mathrm{m} \mathrm{CMOS}$ \\
\hline Supply voltage & $2 \mathrm{~V}$ \\
\hline Power dissipation & $34 \mathrm{~mW}$ \\
\hline Transimpedance gain & $52 \mathrm{~dB} \Omega$ \\
\hline Bandwidth & $3.9 \sim 7.6 \mathrm{GHz}$ \\
\hline Sensitivity & $-19 \mathrm{dBm}$ \\
\hline Chip area & $0.84 \times 0.61 \mathrm{~mm}^{2}$ \\
\hline
\end{tabular}

width ranging from 3.9 to $7.6 \mathrm{GHz}$ with a transimpedance gain of $52 \mathrm{~dB}$. The built-in tuning mechanism provided by the TIA can be used to compensate for the process variation in circuit fabrication. In addition, the TIA operation can also be optimized to achieve the lowest BER in the design of an optical receiver frontend.

\section{REFERENCES}

[1] H. Shigematsu, M. Sato, T. Suzuki, T. Takahashi, K. Imanishi, N. Hara, H. Ohnishi, and Y. Watanabe, "A 49-GHz preamplifier with a transimpedance gain of $52 \mathrm{~dB} \Omega$ using InP HEMTs," IEEE J. Solid-State Circuits, vol. 36, no. 9, pp. 1309-1313, Sep. 2001.

[2] C. Q. Wu, E. A. Sovero, and B. Massey, "40-GHz trans- impedance amplifier with differential outputs using InP-InGaAs heterojunction bipolar transistors," IEEE J. Solid-State Circuits, vol. 38, no. 9, pp. 1518-1523, Sep. 2003.

[3] S. M. Park and H.-J. Yoo, "1.25-Gb/s regulated cascode CMOS transimpedance amplifier for gigabit Ethernet applications," IEEE J. SolidState Circuits, vol. 39, no. 1, pp. 112-121, Jan. 2004.

[4] W.-Z. Chen and R.-M. Gan, "1.8 V, variable gain transimped- ance amplifier with constant damping factor for burst-mode optical receiver," in IEEE RFIC Symp. Dig., Jun. 2005, pp. 12-14.

[5] C. Kromer, G. Sialm, T. Morf, M. L. Schmatz, F. Ellinger, D. Erni, and H. Jackel, "A low-power 20-GHz 52- dB $\Omega$ transimped- ance amplifier in 80-nm CMOS," IEEE J. Solid-State Circuits, vol. 39, no. 6, pp. 885-894, Jun. 2004.

[6] C.-H. Wu, C.-H. Lee, W.-S. Chen, and S.-I. Liu, "CMOS wide- band amplifiers using multiple inductive-series peaking tech- nique," IEEE J. Solid-State Circuits, vol. 40, no. 2, pp. 548-552, Feb. 2005. 